\title{
Microsystems for Three Axis Active Fiber Alignment
}

\author{
Vijay R. Dhuler, Robert L. Wood, John M. Haake*, Allen B. Cowen \\ MEMS Technology Application Center, MCNC \\ 3021 Cornwallis Road, RTP, NC 27709 \\ *The Boeing Company \\ PO Box 516, St. Louis, MO 63166
}

\begin{abstract}
This paper reports the development of novel microsystems capable of three-axis active in-package alignment of fiber optic and micro-optic components with submicron tolerances. Three competing designs have been successfully fabricated and tested. These devices are batch fabricated using a combination of silicon micromachining and LIGA. A novel thermal in-plane actuation mechanism has been developed and used to generate high force and large displacement. High force is necessary to overcome friction, fiber bending, counter force springs, and wire bonds. Displacements exceeding 20 microns have been demonstrated in all three axes in an in-package configuration. Devices were subjected to over 10 million cycles of operation and showed no failure or fatigue.
\end{abstract}

\section{INTRODUCTION}

Single mode optical fibers are expected to bring rapid advancements in a wide array of photonic applications. High bandwidth handling capabilities of single mode fibers is key to enabling high power and high data rate applications such as video links, fiber laser and fiber amplifier systems, and direct-write laser printing. Principle reason for lack of implementation of all optical and single mode opto-electronics in the consumer and military marketplace is the lack of reliable and cost-effective single mode fiber optic alignment and bonding to discrete opto-electronic and photonic devices. The extremely small apertures of single mode fibers make the fiber-to-fiber and fiber-to-photonic connections an exacting and expensive process. Existing procedures for aligning fiber optics and micro-optics are very labor-intensive and require expensive capital equipment. The packaging of individual single mode opto-electronic components currently dominates the product cost in excess of $50 \%$, limiting their implementation in the market place.

Two fundamental approaches are employed in fiber alignment: Active and passive. In active alignment, the operator or the machine manipulates the fiber relative to the coupled device and seeks active feedback, such as the output from a photodiode, to optimize the alignment. Once optimized, the fiber is cemented in place using adhesive or eutectic bonding. The existing procedures for active alignment are very expensive. Passive alignment relies on precision fixturing of the fiber relative to coupled device. A typical example is the use of a micromachined $\mathrm{V}$-groove in a silicon substrate for precision placement of the fiber followed by bonding. Silicon micromachining permits very precise definition of the $\mathrm{V}$ groove with respect to the fiber dimensions and to the silicon surface, which in turn can provide accurate one-dimensional fixturing of fibers. The passive alignment approach is potentially much more cost effective than active alignment, yet it has not measured up to the demands for 3-axis alignment. Researchers [1, 2] have reported microfabricated active alignment strategies that are either not fully integrated, or do not provide alignment in all three axes.

\section{DESIGN AND FABRICATION}

Three different microsystem designs [3] (Figs. 1-3) have been fabricated and tested. Each design provides a platform for holding the fiber using either a V-Groove or a LIGA defined channel. The platform is moved in three axes with respect to a fixed reference provided either internally or externally to the silicon chip. Novel inplane thermal actuators located either on the movable platform or on the fixed base move the platform and hence the fiber in two axes. A thermal bimorph actuator lifts the platform out-of-plane. The forces generated by the actuators are large enough to overcome the friction between the moving and the fixed surfaces, the restoring force provided by the fiber and the wire bonds, and the counter force springs. These actuators provide displacements in excess of twenty microns, which significantly reduces the required pre-alignment accuracy. Also, note that the designs presented here have not yet been optimized for the size. We believe that the sizes could be reduced significantly.

\section{Design 1}

Figure 1 shows the plan and cross-sectional schematic of design 1 . The end of the fiber to be aligned is glued in the V-groove provided on the silicon platform. The silicon platform is mounted onto an external base using two fixed mounts on the external base and the LIGA frame provided on the platform. The LIGA frame has two sets of four arms that fit onto the mounts. The mounts pass through the holes fabricated in the silicon platform. The LIGA frame is supported on the silicon platform by four LIGA springs and two anchors. This arrangement allows for three axis motion of the silicon platform with respect to the fixed mounts and the external base. The fixed mounts and the external base serve as the reference for alignment. This first generation design as depicted does not support out-of-plane (third axis) motion. $\mathrm{X}$ and $\mathrm{Y}$ actuators for in-plane motion are attached to the silicon platform and push against the LIGA frame to move the silicon platform in two axes. The photonic device to be aligned to the fiber would be mounted on the external base.

\section{Design 2}

Figure 2 shows a three dimensional schematic of design 2 . The end of the fiber to be aligned is glued in the V-groove provided on the silicon platform. The silicon platform is housed in an external housing. Two LIGA counter force springs are provided on two sides of the platform and fixed LIGA bearings on the other two sides. They extend beyond the edge of the silicon platform and define the periphery of the platform accurately. This allows for accurate prealignment of the fiber with respect to the external housing. $X$ and $Y$ 
actuators for in-plane motion are attached to the silicon platform and have arms extending beyond the edge of the platform. These arms push against the reference wall of the external housing and move the platform in two axes against the counter force springs.

In addition to the in-plane actuators an out-of-plane actuator is fabricated on the silicon platform. This actuator is a thermal bimorph of single crystal silicon and LIGA-defined Nickel. When actuated it lifts the silicon platform out-of-plane by acting against the base of the external housing. The photonic device that needs to be aligned is mounted into the external housing.

\section{Design 3}

Figure 3 shows a three dimensional schematic of design 3. In this design the silicon platform is divided into two sections: a fixed mounting base and a movable base. The end of the fiber to be aligned is glued in the LIGA-defined fiber optic channel provided on the movable base, which is free to move both in plane and outof-plane. The movable base is supported by four LIGA fabricated counter force and retainer springs, which are anchored to the fixed mounting base. $\mathrm{X}$ and $\mathrm{Y}$ actuators for in-plane motion are attached to the fixed mounting base. When actuated they push against the fixed LIGA surfaces on the movable base moving it in two axes

In this case, the out-of-plane actuator, which is a thermal bimorph similar to that used in design 2 , is fabricated on the movable base. When actuated it lifts the movable base by acting against the surface on which the platform is supported. The photonic device that needs to be aligned is mounted on the same surface on which the silicon platform is mounted.

Design 3 is the most compact of the three designs and requires the least amount of assembly and pre-alignment. It is also the least expensive of the three designs.

\section{Fabrication}

The silicon platforms in all the three designs are fabricated using a similar process. Fabrication starts with a single crystal silicon substrate. Surface micromachining and LIGA techniques are used to define the actuators and the various springs. Anisotropic etching is used to define the V-groove, out-of-plane actuator, separation channel for design 3 and holes for design 1 .

\section{RESULTS AND DISCUSSION}

To characterize the performance of these actuators and microsystems, an inexpensive but accurate method of measuring force and displacement by using a calibrated, cantilevered fiber optic beam as a force probe has been developed. This method has a large dynamic range from $10 \mu \mathrm{N}$ to 100 's of $\mathrm{mN}$ with accuracies of a few percent $[4,5]$.

\section{Displacement Measurement}

Displacement measurements are done using a high power microscope with a translation stage and a graduated microscope eyepiece. The translation stage is equipped with an electronic vernier for $\mathrm{x}, \mathrm{y}, \mathrm{z}$ motion with an accuracy of $+/-1$ micron.

As shown in figure 4 the displacement is fairly linear as a function of the applied power. Displacements of up to 30 microns are realized for an applied power of $0.45 \mathrm{~W}$. Qualitative characterization of the out-of-plane actuator indicates platform displacements in excess of 100 microns for powers less than 0.5 watts.

\section{Force Measurement}

The measurement of force involves the accurate measurement of the fiber optic force probe base deflection and the movement of the actuator to which the fiber optic force probe is being applied $[4,5]$. By measuring the fiber optic probe tip deflection the applied force to the actuators is calculated. There is a limit to how much force could be applied to the actuator before buckling occurs. Therefore, a small displacement on the actuator of 5 microns in the direction opposite to that of the actuator movement was used to measure the force that an actuator can exert at different power levels. Figure 5 shows a plot of force as a function of power applied to the actuator. The plot indicates that the actuators can supply a force of $\sim 20 \mathrm{mN}$ at 0.3 watts.

\section{Dynamic Characterization}

A laser vibrometer was used to measure the rate at which the platform could move axially. Figure 6 shows the plot of the laser vibrometer output as a function of the frequency of oscillation. The plot indicates a $3 \mathrm{~dB}$ point at $60 \mathrm{~Hz}$.

\section{In Package Demonstration}

Figure 7 shows the experimental setup and demonstration for twoaxis active alignment for design 1 in an in-package configuration. The lateral actuator is used to bring the actuated fiber in alignment with the fixed fiber. The second in-plane actuator is used for focusing. Sinusoidal actuation signals are applied to the actuators. Oscilloscope display of the drive and the detected signals indicates the alignment of the fibers. Design 3 has been successfully tested for three axis motion of the platform.

\section{CONCLUSIONS}

The design, fabrication, and operation of microsystems for active three axis fiber alignment have been demonstrated. Thermal actuator technology capable of large force $(>20 \mathrm{mN})$ and displacements $(>20 \mu \mathrm{M})$ has been implemented. Operation cycles in excess of 10 million cycles have been demonstrated.

\section{ACKNOWLEDGMENT}

This work was performed under Army Research Office contract funded by the DARPA-Defense Sciences Office. The authors wish to thank Dr. Robert Crowe of DARPA-DSO and Dr. John Prater of ARO for their support.

\section{REFERENCES}

1. Li Fan, et al., "Self-Assembled Microactuated XYZ stages for Optical Scanning and Alignment", Transducers 97 Technical Digest, Vol. 1, p. 319.

2. R. Jebens, et al., "Microactuators for Aligning Optical Fibers", Sensors and Actuators, 20 1989, p. 65.

3. Patenting in progress

4. J. Haake, et al., "In-Package Active Fiber Optic Micro-Aligner", Photonics West 98 Technical Digest.

5. J. Haake et al., "Direct Measurement of Force and Displacement of LIGA Micro Springs and Actuators", Technical Digest, SPIE 1997 Conference on Micromachining and Microfabrication. 


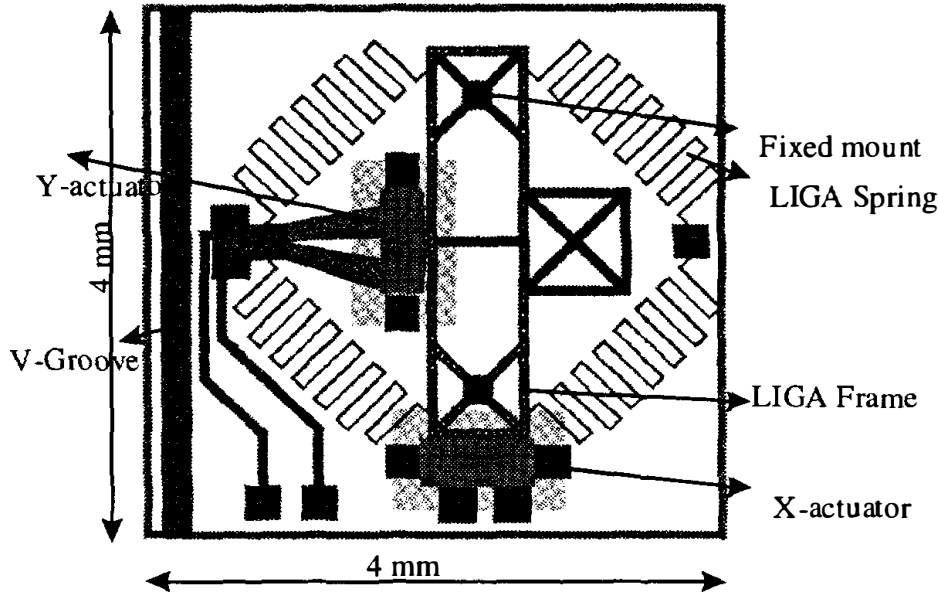

(a)

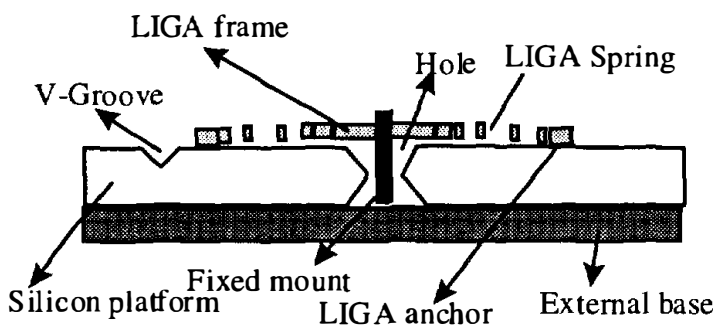

(b)

Figure 1. 2-axis In-package micro aligner (Design 1): (a) plan view schematic; (b) cross-sectional schematic

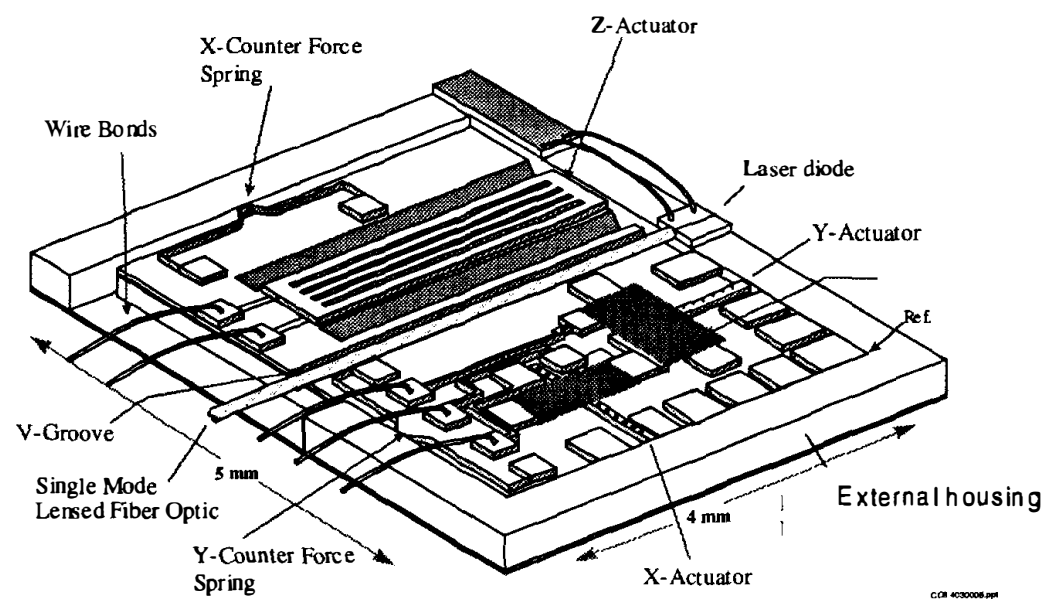

Figure 2. 3-axis In-package micro aligner (Design 2)

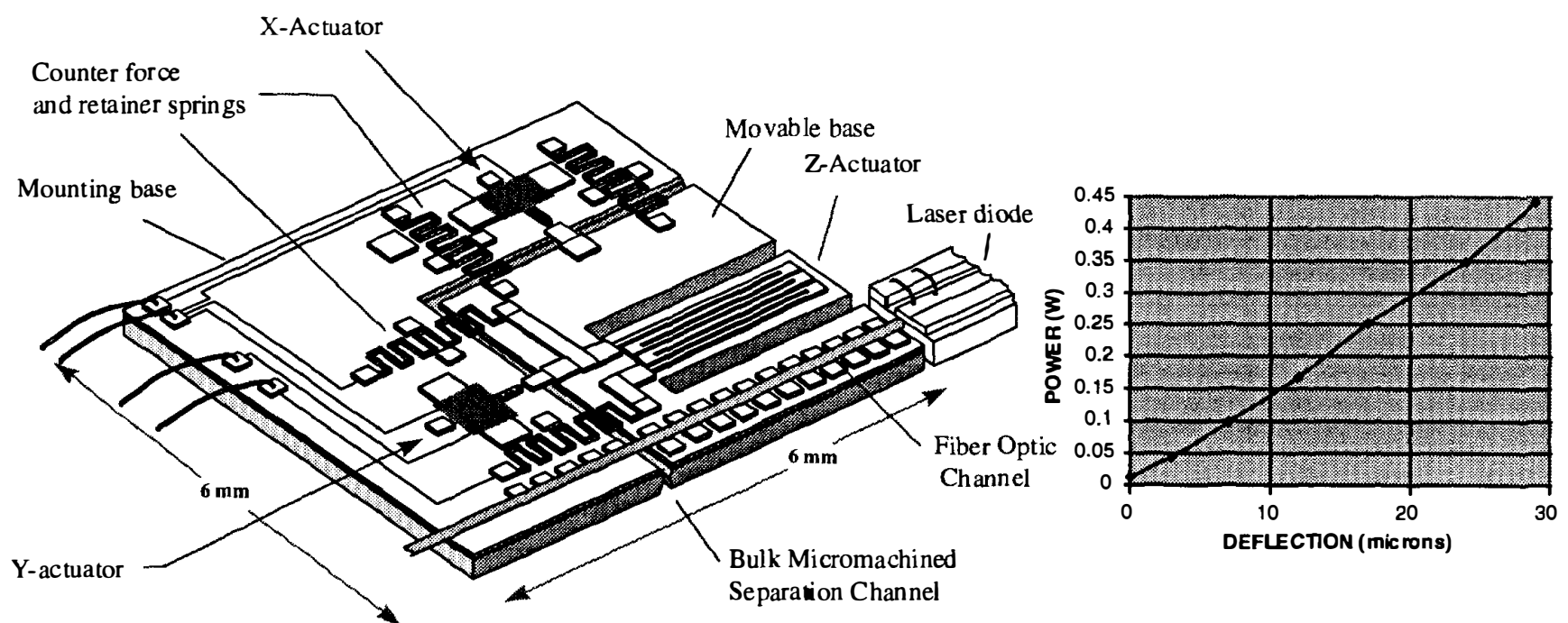

Figure 3. Mounted 3-axis in-package micro aligner (Design 3)

Figure 4. In-plane thermal actuation 
* 240 micron Diameter Polyimide Coated fiberoptic

* Fiber optic Length $=11 \mathrm{~mm}$

* Forced measured at $5 \mathrm{Hm}$ back deflection of actuator at each power level $-3 \mathrm{~dB} @ 60 \mathrm{~Hz}$
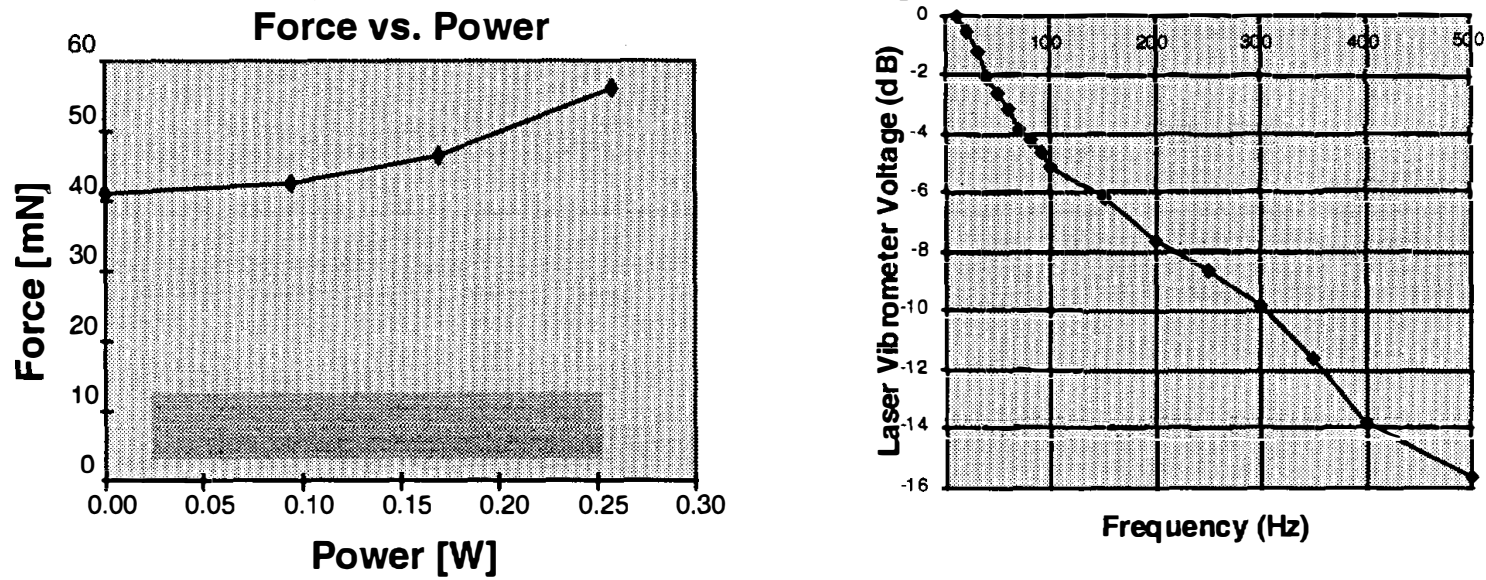

Frequency $(\mathrm{Hz})$

Figure 5. In-plane actuation: force versus power

Figure 6. Mechanical frequency of in-plane actuation (design 1)

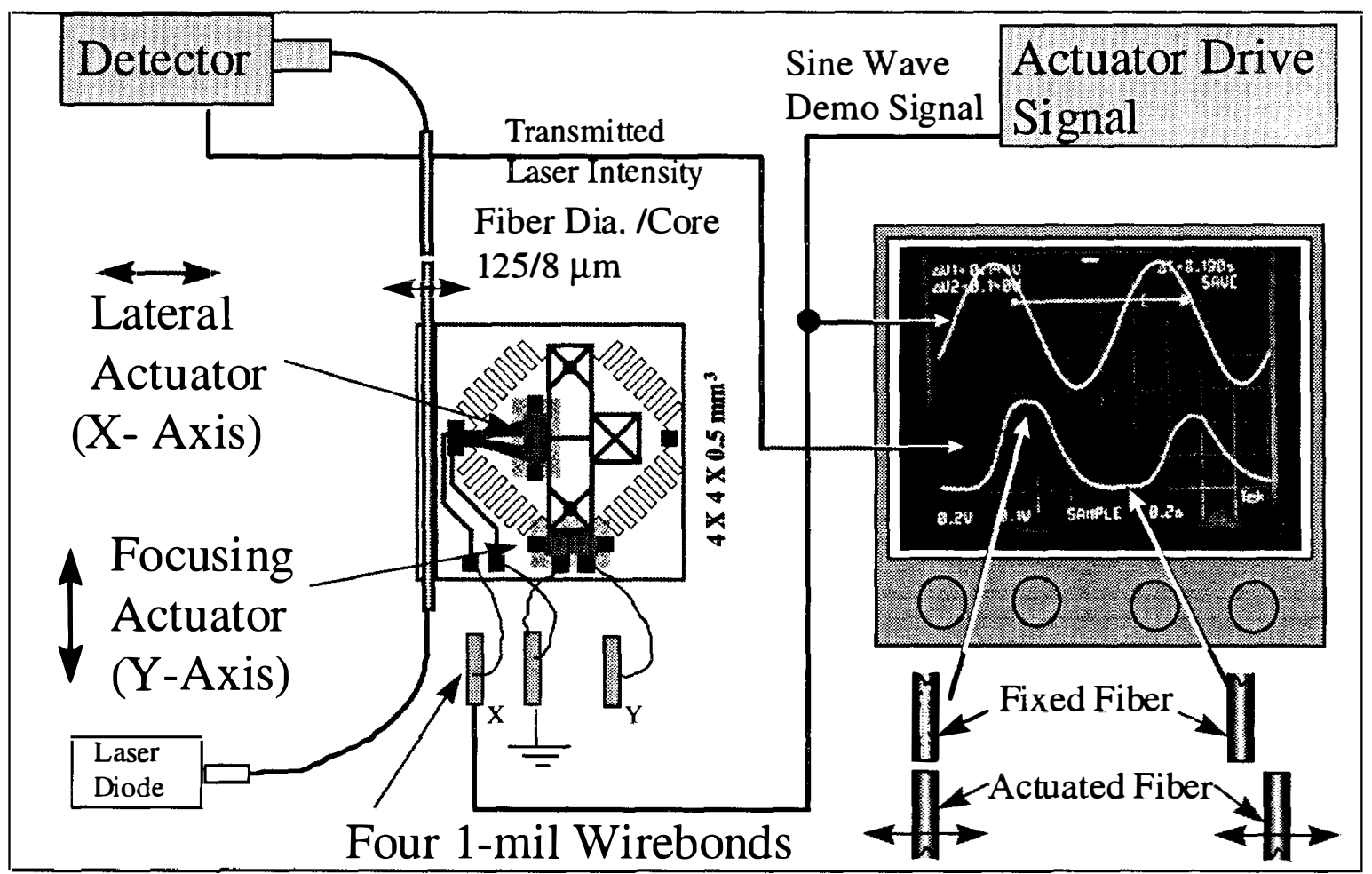

Figure 7. Two-Axis active fiber aligner experimental set-up and demonstration 